\title{
Crystallization of Filled Nylon 6. I. Heat Capacities and Melting Behavior
}

\author{
V. P. Privalko, T. KaWAi, and Yu. S. Lipatov \\ Institute of Macromolecular Chemistry, Academy of Sciences of the Ukrainian \\ SSR, 252160, Kiev, USSR, and Department of Polymer Technology, Tokyo Institute \\ of Technology, Ookayama, Meguro-ku, Tokyo 152, Japan.
}

(Received January 29, 1979)

\begin{abstract}
The influence of untreated glass beads (GB) and Aerosil (A), as well as glass beads treated with aminosilanes (GBA) on the melting behavior of nylon 6 was studied with the aid of differential scanning calorimeter (DSC-1). It was found that glass beads with low surface energy (GBA) exerted no appreciable influence on the melting characteristics of a polymer, while in filled samples of the GB and A series, heats of crystallization and melting linearly decreased with a total solid surface area, the slope being numerically equal to the heat of the wetting of A by nylon 6 melt. Equilibrium melting temperature in the samples of these series abruptly dropped from $511 \mathrm{~K}$ to $499 \mathrm{~K}$ after interparticle separation decreased below about $x=10 \times 10^{-6} \mathrm{~m}$, and remained thereafter approximately constant at $T_{\mathrm{m}}{ }^{0}=501 \pm 3 \mathrm{~K}$ down to $x=50 \times 10^{-6} \mathrm{~m}$. At $x=30 \times 10^{-6} \mathrm{~m}$, no crystallization was observed above $440 \mathrm{~K}$, and the melting temperature of this sample when cooled to room temperature was independent of the crystallization temperature in the range below $440 \mathrm{~K}$. Annealing of these samples at $300 \mathrm{~K}$ resulted in the appearance of relaxation endotherms on the heat capacity curves above the glass-transition interval, the strength of said endotherms smoothly increasing with the filler content. The experimental data were discussed in terms of changes in the initial melt structure of nylon 6 and competition between surface forces tending to increase the filler coverage and opposing its bulk driving force for polymer crystallization tending to pull segments from filler surface onto growing crystal face.
\end{abstract}

KEY WORDS Crystallization / Heat Capacity / Melting Behavior / Nylon 6 / Filler / Inter Particle Separation /

Incorporation of inorganic fillers into organicpolymer matrixes was originally for the purpose of acheiving optimum mechanical properties in the resulting composites by combining the high modulus and strength of the fomer with the elasticity of the latter. However, it was found that not only mechanical but also many other static and dynamic properties of filled polymers invariably failed to obey the simple linear rule of mixtures, the magnitude (and in some cases even the sign) of the deviations from additivity being strongly dependent both on the chemical nature of components, as well as on their relative content. ${ }^{1-8}$ These manifestations of the "long-range" nature of the solid-surface effect on polymer structure and properties were attributed to the formation of boundary-polymer layers with changed properties as a result of a complex interplay of energetic and entropic phenomena involved in the polymer-filler interactions. ${ }^{9}$ The applicability of this concept, which has proved successful for interpreting the bulk properties of filled amorphous polymers, ${ }^{10-14}$ in the case of filled crystallizable polymers was, however, tested only on a relatively modest scale, ${ }^{15-17}$ since so far the main attention of polymer scientists has been directed toward investigating crystal-nucleation phenomena in polymer melts containing very small amounts of solid particles. ${ }^{18-25}$ In the present series of papers, we discuss the results of an extensive experimental study of crystallization and melting behavior of nylon 6 containing various amounts of silica and glass beads, including the range of filler loadings where polymer properties were expected to be most subject to solidsurface influence.

\section{EXPERIMENTAL}

Nylon 6 resin, used throughout this study, was a 
commercial product of Toyo Rayon Co. Ltd. Two kinds of silica-based glass beads (GB 731 and GB $731 \mathrm{~A}$ series produced by Toshiba-Barotini Co. Ltd.) and fine silica powder (Aerosil 200, product of Nihon Aerosil Co. Ltd.) were used as fillers. Both types of glass beads had the same chemical composition $(72 \%$ $\left.\mathrm{SiO}_{2}\right)$, equal densities $\left(0.248 \mathrm{~kg} \mathrm{~m}^{-3}\right)$, and mean sizes (about $3 \times 10^{-5} \mathrm{~m}$ ), but the series GB $731 \mathrm{~A}$ was surface-treated with a aminosilane coupling-agent. Untreated silica powder had essentially the same chemical composition as glass beads $\left(99 \% \mathrm{SiO}_{2}\right)$, but a much lower mean particle size $\left(1.2 \times 10^{-8} \mathrm{~m}\right)$ and, correspondingly, a higher specific surface area $\left(S=2 \times 10^{5} \mathrm{~m}^{2} \mathrm{~kg}^{-1}\right)$. Prior to use, all fillers were washed with methanol and vacuum-dried for 8 hours at room temperature. The following procedure was adopted for preparing the filled samples. The polymer was first refluxed with methanol, dried and then dissolved in anhydrous formic acid (polymer concentration, $2-3 \%$ ), after which the necessary amount of filler was added to solution. This treatment not only ensured favorable conditions for homogeneous distribution of filler particles in polymer matrix, but served also to stabilize the molecular weight of the latter during melting-cystallization cycles. $^{26,27}$ The solvent was subsequently slowly evaporated overnight under conditions of mild warming (to about $310 \mathrm{~K}$ ) and periodical stirring, and then evacuated for 8 hours at room temperature. Precipitates obtained at this stage were then crushed to fine powder and evacuated for another 3 days to remove any traces of residual solvent. The powdered, solvent-free samples were hot-pressed at $500-510 \mathrm{~K}$ for 15 min to obtain homogeneous films from which samples were cut for calorimetric measurements. Filled samples were labelled according to the filler type and its weight content, e.g., GB-50 (50 wt percent of GB 731); GBA-60 (60 wt percent of GB 731A), or A-3 (3 wt percent of Aerosil 200). The weight of a filled film was varied to match approximately the weight of its polymer component by using the weight of a pure-polymer sample (about $1 \times 10^{-5} \mathrm{~kg}$ ). All calorimetric work reported in this series was carried out using the DSC-1 instrument (Perkin-Elmer Corp.). The temperature and heat of the phase transitions were calibrated using variable amounts of bismuth, tin, and indium for hightemperature measurements, and distilled water for lower temperature measurement, at scan speeds ranging from $1-64 \mathrm{~K} \mathrm{~min}^{-1}$ and at different settings of instrumental sensitivity. For heat-capacity determinations, the instrument was calibrated and periodically checked in the range $200-600 \mathrm{~K}$ according to standard procedures, ${ }^{28,29}$ using sapphire as the standard. All heat-capacity measurements were performed in a flux of dry nitrogen at a scanning speed $16 \mathrm{~K} \mathrm{~min}^{-1}$. The estimated errors in temperature, heats of transition, and heat capacities were $1 \mathrm{~K}, 5 \mathrm{~kJ} \mathrm{~kg}^{-1}$ and $25 \times 10^{-3} \mathrm{~kJ} \mathrm{~kg}^{-1} \mathrm{~K}^{-1}$, respectively. To ensure reproducible and comparable experimental conditions, all samples prior to measurement were kept for $5 \mathrm{~min}$ at $550 \mathrm{~K}$.

\section{RESULTS}

\section{Heat Capacities}

As can be seen from Figure 1, the overall shapes of the heat capacity, $c_{p} v s$. temperature, $T$ curves for all samples which were rapidly ( 32 to $64 \mathrm{~K} \mathrm{~min}^{-1}$ ) cooled from the melt (hereafter referred to as quenched samples), are typical for semicrystalline polymers, consisting of linear portions below the glass-transition temperature, $T_{\mathrm{g}}$ (observable as a break in the vicinity of $325 \mathrm{~K}$ ), and above the experimental melting point, $T_{\mathrm{m}}$ (defined as a highesttemperature peak on the melting endotherm), and of a nonlinear portion in the intermediate temperature range. Heat capacities of unfilled nylon 6 both in the glassy state below $T_{\mathrm{g}}$, and in the molten state above $T_{\mathrm{m}}$, agreed to within $2 \%$ with the corresponding data of Gee et al. ${ }^{30} c_{p}$ 's of filled samples were found to decrease linearly with increasing filler weight content, $W$, from $c_{p}$ (polymer) to $c_{p}$ (filler) both in the melt, as well as in the temperature range below $T_{\mathrm{g}}$ for quenched samples (upper and lower lines in Figure 2). Deviations from additivity did not generally exceed the limits of experimental uncertainty. Heat capacities of all fillers used in the present study agreed to $3 \%$ with the following empirical equation derived from our experimental data in the temperature range from 220 to $550 \mathrm{~K}$ (lowest curve in figure 1):

$$
c_{p}=0.033+3.24 \times 10^{-3} T-2.56 \times 10^{-6} T^{2}
$$

with $c_{p}$ in $\mathrm{kJ} \mathrm{kg}^{-1} \mathrm{~K}^{-1}$ and $T$ in $\mathrm{K}$. The location of the $T_{\mathrm{g}}$ interval did not reveal systematic dipendence on $W$ and remained essentially unchanged in the relatively narrow range $320-330 \mathrm{~K}$. On the other hand, both absolute values, as well as a general pattern of $c_{p}=f(T)$ plots in the vicinity of and above 


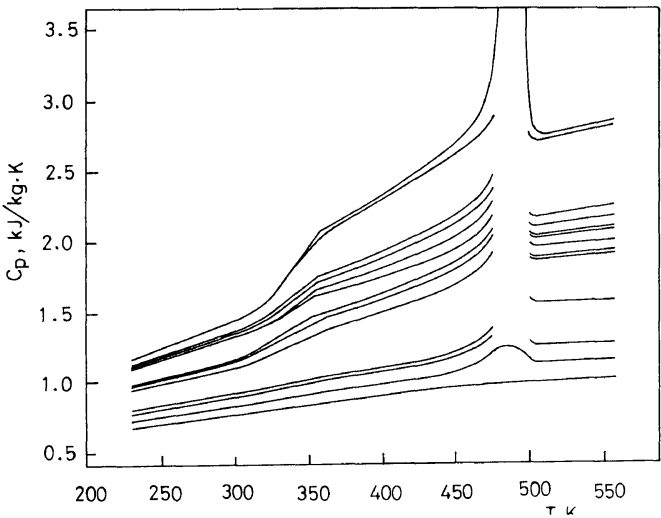

Figure 1. Temperature dependence of heat capacity for quenched samples of (from top down); pure nylon 6; A-3; A-20; A-33; GB-50; A-50; GBA-60; A-67; GB-70; GBA80; GB-80; GB-83; glass beads and aerosil.

$T_{\mathrm{g}}$, were found to be markedly time-dependent in cases of samples containing untreated fillers (series GB and A), whereas only minor changes could be detected for either pure polymer, or filled GBA series. One can see from Figure 3 that 4-days storage of quenched samples from GB and A series at $300 \mathrm{~K}$ resulted in the appearance of a first-order transitionlike endotherm with its maximum located some 30 $50 \mathrm{~K}$ above $T_{\mathrm{g}}$. Although quantitative results were not obtained at the present stage due to an extremely high time-dependence of "dynamic" $c_{p}$ data in this temperature range, one can still draw a broad conclusion that the apparent "heat of transition" calculated per pure polymer, smoothly increases with filler content $W$ (or, rather, with a total solidsurface available, $S W /(1-W))$. It is noteworthy too, that the endothermic peak referred to above was completely absent on the thermograms of samples heated to only $380-400 \mathrm{~K}$, cooled to below $T_{\mathrm{g}}$ and immediately heated again, while it reappeared again after prolonged storage at room temperature. No evidence of a time-dependent heat capacity above $T_{\mathrm{m}}$ was observed, even for samples of GB and A series with the highest filler content.

\section{Melting Behavior}

In conformity with earlier studies, ${ }^{31-33}$ at least 3 peaks could be detected on the melting endotherm of pure nylon 6 isothermally crystallized from the melt in a temperature range from 440 to $480 \mathrm{~K}$ (Figure 4a). As can be seen from Figure 5a, temperatures

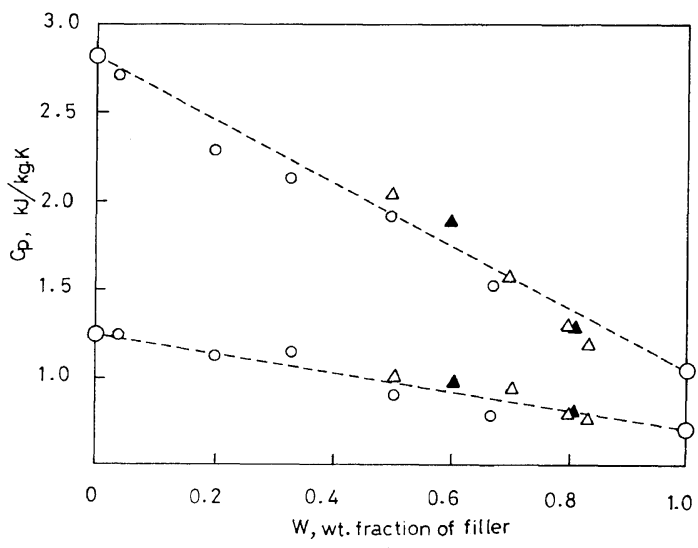

Figure 2. Composition dependence of heat capacity above $T_{\mathrm{m}}$ (upper line) and below $T_{\mathrm{g}}$ (lower line) of filled samples of series A (open circles), GB (open triangles), and GBA (filled triangles).

corresponding to the occurrence of all these peaks, $T_{\mathrm{m}}(i)$ (where $i$ is the peak number as defined in Figure 4 ), as well as melting temperature of a sample isothermally crystallized to only $5-10 \%$ of a total degree of transformation ${ }^{34,35}$ (line 4 in Figure 5a), exhibited essentially linear dependence on crystallization temperature, $T_{\mathrm{c}}$, at least in a restricted temperature interval. Numerical values of empirical parameters $a(i)$ and $b(i)$ from equation

$$
T_{\mathrm{m}}(i)=a(i)+b(i) T_{\mathrm{c}}
$$

describing these straight lines, are listed in the table. The results obtained show that the slope $b(i)$ decreases from about unity for line 1 to 0.5 for lines 2 and 4, and becomes zero for line 3 . The overall shape of the melting endotherm remained essentially unchanged when GBA filler was added to initial nylon 6 sample, whereas melting peaks were found to become gradually less sharp with increasing filler content in filled GB and A series ( $c f$. typical thermograms in Figures $4 \mathrm{~b}$ and $4 \mathrm{c}$ ). As can be seen from Figures $5 \mathrm{~b}$ and $5 \mathrm{c}$, as well as from the numerical data in the table, parameters of eq 1 for filled samples of GBA series are not appreciably different from those of a pure polymer. On the other hand, while slope $b(1)$ was nearly unity for all filled samples independent of their composition, both slopes $b(2)$ and $b(4)$, and intercepts $a(2)$ and $a(4)$, underwent marked changes due to the addition of untreated GB and A fillers. The melting behavior of sample A-67 was extraordinary in the sense that its melting endotherm 

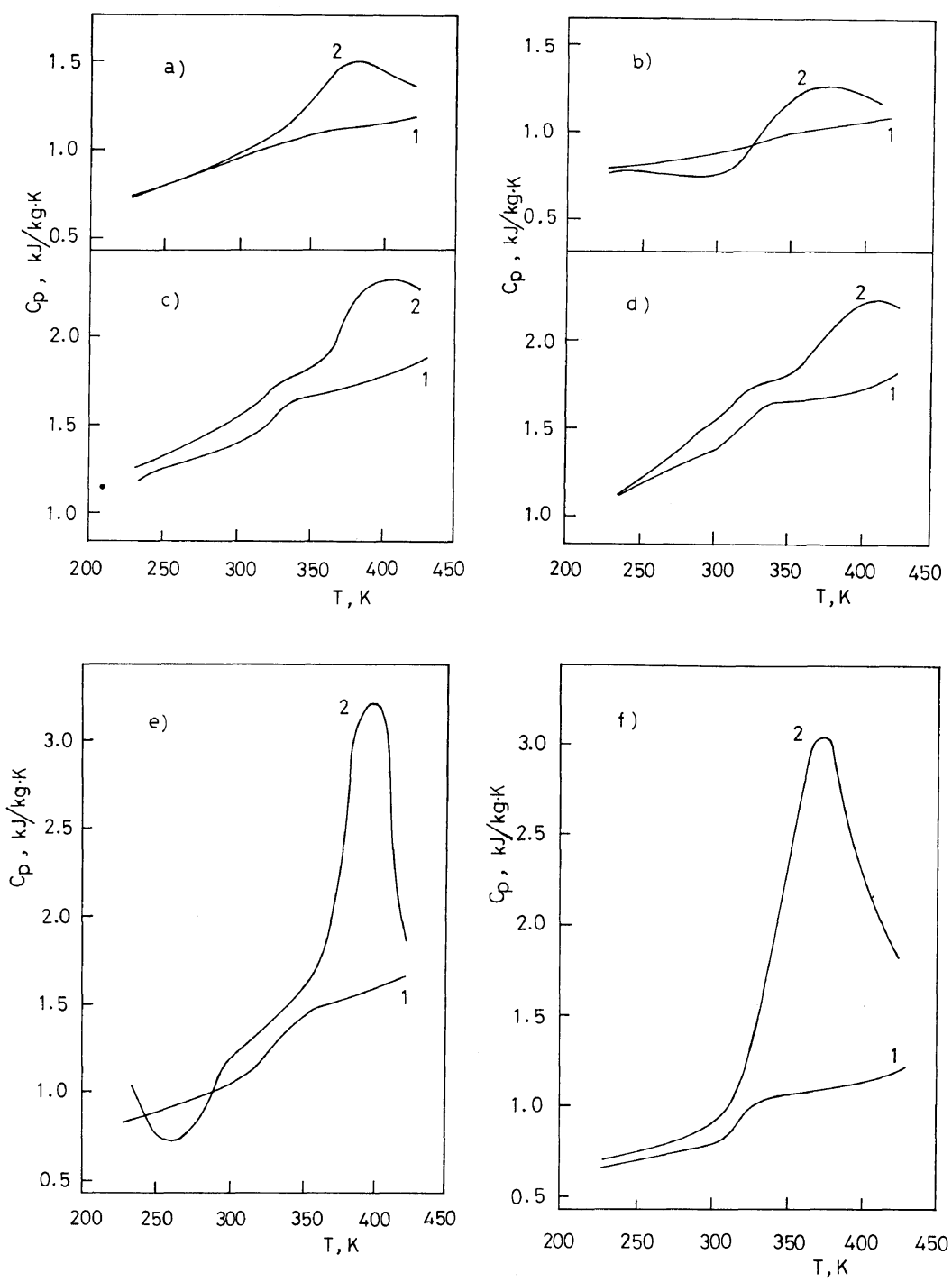

Figure 3. Low-temperature heat capacity of quenched (curves 1) and annealed (curves 2) samples GB-80 (a), GB-83 (b), A-20 (c), A-33 (d), A-50 (e), and A-67 (f).

consisted of only one diffuse peak whose temperature was independent of $T_{\mathrm{c}}$ and remained virtually constant at $T_{\mathrm{m}}=478 \pm 2 \mathrm{~K}$.

Crystallization and melting enthalpy of isothermally crystallized, pure nylon 6 increased from $\Delta H_{\mathrm{m}}{ }^{*}=47.3 \mathrm{~kJ} \mathrm{~kg}^{-1} \quad$ at $\quad T_{\mathrm{c}}=441.2 \mathrm{~K} \quad$ to $\Delta H_{\mathrm{m}}{ }^{*}=59.1 \mathrm{~kJ} \mathrm{~kg}^{-1}$ at $T_{\mathrm{c}}=476.1 \mathrm{~K}$, which corresponds to an increase of crystallinity from $X=0.27$ to $X=0.34$, assuming $\Delta H_{\mathrm{m}}=176.4 \mathrm{~kJ} \mathrm{~kg}^{-1}$ for melting enthalpy of a perfect nylon 6 crystal. ${ }^{30}$ Similar data were reported by Illers et al. ${ }^{31}$ and Valenti et $a l^{33}$ On the other hand, while essentially the same trend was observed for filled samples of GBA series, samples containing large amounts of untreated GB and A fillers exhibited exactly the opposite behavior (see Table I of a companion paper, ref 36). Moreover, it was also noted that heats of crystallization and melting of isothermally crystallized samples of the latter series systematically decreased with increasing filler content, until no crystallization 

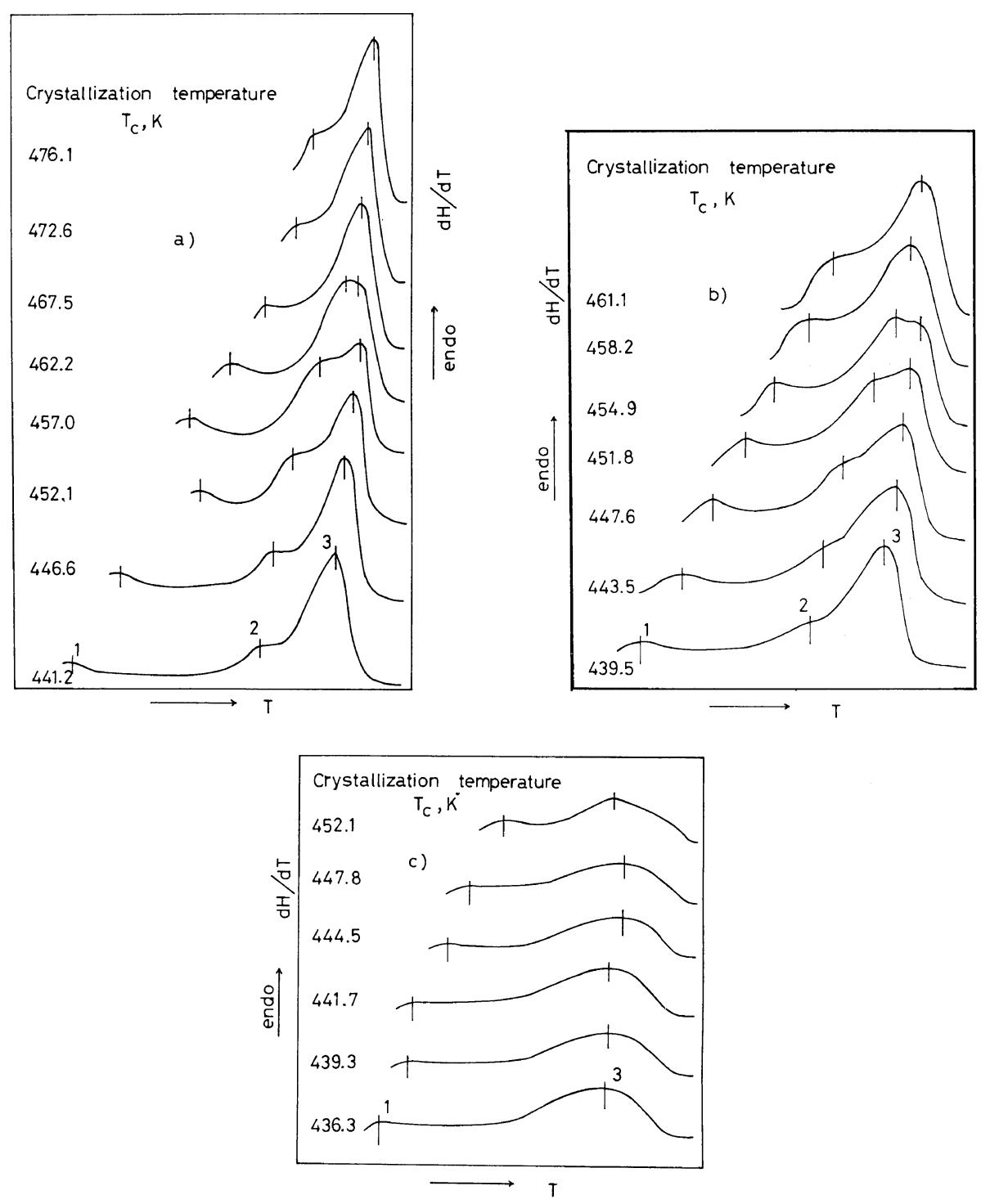

Figure 4. Melting endotherms of pure nylon 6 (a), and filled samples A-20 (b) and A-50 (c) isothermally crystallized at indicated temperatures.

exotherm at all could be detected for sample A-67. Closely related phenomena were found by Howard ${ }^{16}$ for poly(ethylene oxide) containing increasing amounts of untreated Aerosil. As can be seen from Figure 6 (line 1), $\Delta H_{\mathrm{m}}{ }^{*}$ is found to be an approximately linear function of the total solid surface area, $S W /(1-W)$, as expressed by the following equation

$$
\Delta H_{\mathrm{m}}^{*}=a^{\prime}+b^{\prime} S W /(1-W)
$$

with $a^{\prime}=53 \mathrm{~kJ} \mathrm{~kg}^{-1}$ and $b^{\prime}=185 \times 10^{-3} \mathrm{~J} \mathrm{~m}^{-2}$. Equation 2 was also found to hold reasonably well for samples of the GB and A series which were allowed to cool to room temperature after completion of isothermal crystallization from the melt (line 2 in Figure 6), although in this case, numerical parameter values changed to $a^{\prime}=59 \mathrm{~kJ} \mathrm{~kg}^{-1}$ and $b^{\prime}=65 \times 10^{-3} \mathrm{~J} \mathrm{~m}^{-2}$. 

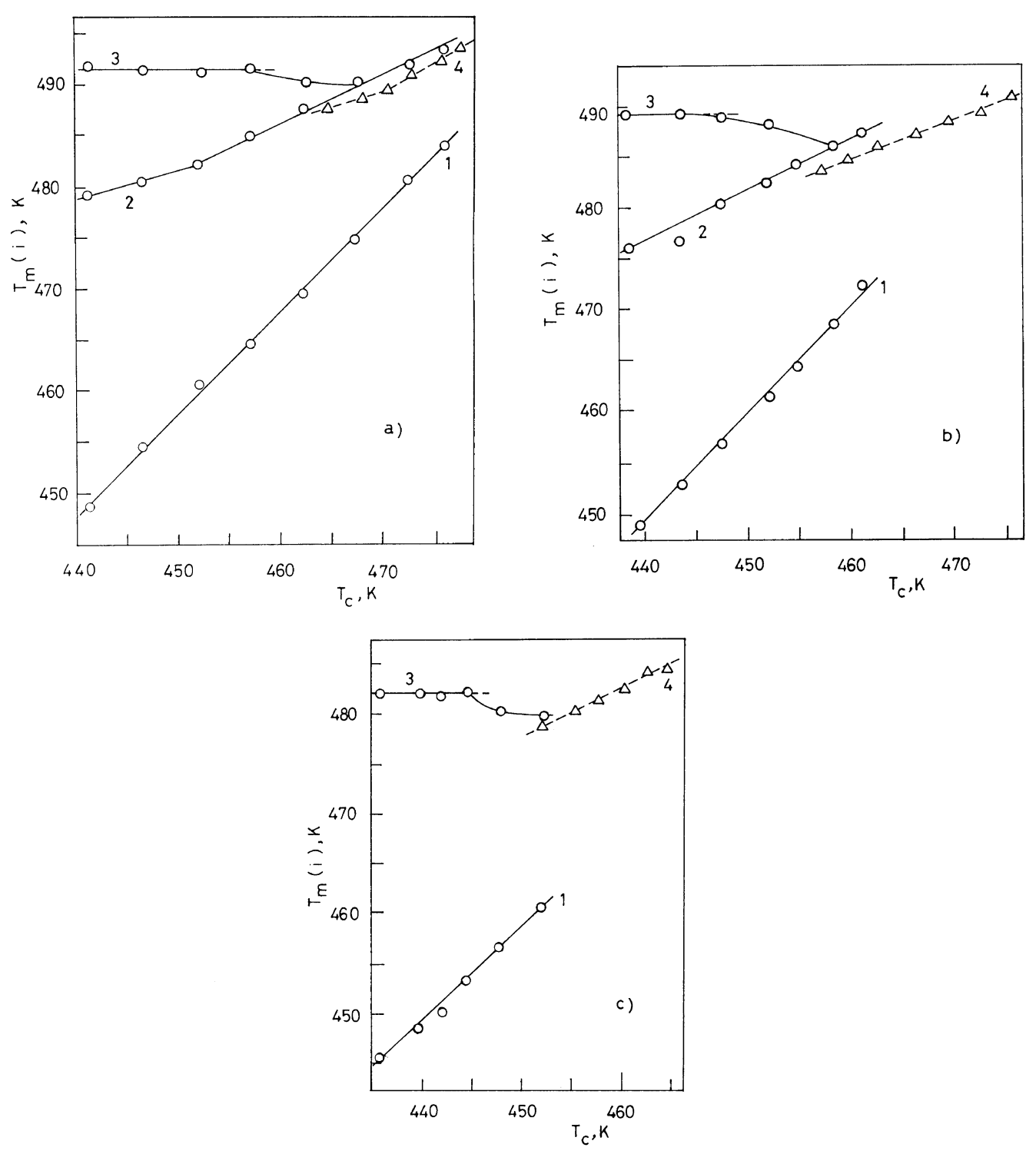

Figure 5. Dependence of melting peaks $T_{\mathrm{m}}(i)$ on crystallization temperature $T_{\mathrm{c}}$ for pure nylon 6 (a), and filled samples A-20 (b) and A-50 (c).

\section{DISCUSSION}

In principle, the occurrence of multiple peaks on the melting endotherms of polymers can be attributed to recrystallization phenomena in the course of heating, ${ }^{37,38}$ successive, step-like melting of folded-chain and bundle-chain lamellae, ${ }^{38,39}$ polymorphic transitions, ${ }^{40}$ etc. To facilitate the structural interpretation of various endothermic processes during melting of our samples, we recall that both $\alpha$ - and $\gamma$-crystalline modifications are formed during melt crystallization of nylon 6 in a temperature range below $450 \mathrm{~K}$, whereas at higher temperatures nylon 6 crystallizes essentially in the $\alpha$-form. ${ }^{31,32,41,42}$ Thus, the initial constancy of $T_{\mathrm{m}}(3)$ and the gradual disappearance of peak 3 when $T_{\mathrm{c}}$ exceeds $455 \mathrm{~K}$ (line 
Crystallization of Filled Nylon 6

Table I. Parameters of eq 1 for systems studied

\begin{tabular}{|c|c|c|c|c|c|c|c|c|c|}
\hline \multirow{2}{*}{ Sample code } & $a(1)$ & \multirow{2}{*}{$b(1)$} & $a(2)$ & \multirow{2}{*}{$b(2)$} & $a(3)$ & \multirow{2}{*}{$b(3)$} & $a(4)$ & \multirow{2}{*}{$b(4)$} & \multirow{2}{*}{$T_{\mathrm{m}}^{0}( \pm 2) / \mathrm{K}$} \\
\hline & K & & $\mathrm{K}$ & & $\mathrm{K}$ & & $\mathrm{K}$ & & \\
\hline Pure polymer & 12.4 & 0.99 & 292.7 & 0.420 & 491.5 & 0 & 236.1 & 0.538 & 511 \\
\hline GBA-60 & -6.7 & 1.03 & 269.1 & 0.470 & 490.7 & 0 & 219.9 & 0.569 & 510 \\
\hline GBA- 80 & 3.8 & 1.01 & 268.8 & 0.470 & 490.6 & 0 & 233.1 & 0.542 & 509 \\
\hline GB-50 & 21.9 & 0.97 & 301.5 & 0.400 & 490.8 & 0 & 255.0 & 0.502 & 512 \\
\hline GB-70 & 7.0 & 1.00 & 268.4 & 0.467 & 488.4 & 0 & 285.5 & 0.428 & $499(498)^{\mathrm{a}}$ \\
\hline GB- 80 & -11.4 & 1.04 & 269.5 & 0.472 & 492.8 & 0 & 302.1 & 0.396 & 500 \\
\hline GB-83 & -26.7 & 1.08 & 349.3 & 0.301 & 495.2 & 0 & 329.0 & 0.347 & 504 \\
\hline A-3 & -13.2 & 1.06 & 335.8 & 0.327 & 491.2 & 0 & 327.1 & 0.345 & 499 \\
\hline A-20 & -11.3 & 1.05 & 267.6 & 0.476 & 489.1 & 0 & 311.1 & 0.378 & 500 \\
\hline A-33 & 11.0 & 1.00 & 272.8 & 0.465 & 488.7 & 0 & 300.9 & 0.399 & 502 \\
\hline A-50 & 9.0 & 1.00 & - & - & 481.9 & 0 & 285.0 & 0.429 & 499 \\
\hline A-67 & - & - & - & - & - & - & 478.0 & 0 & - \\
\hline
\end{tabular}

a This value was obtained in a reproducibility test for a different batch of GB-70.

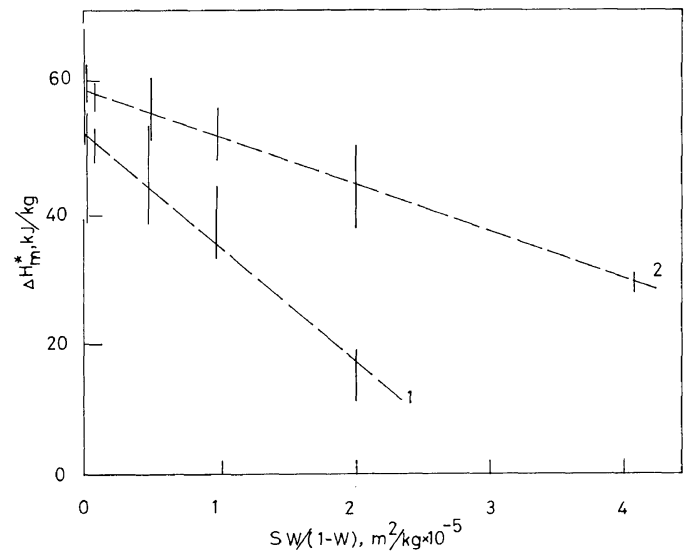

Figure 6. Dependence of crystallization and melting enthalpies $\Delta H_{\mathrm{m}}$ of isothermally crystallized (line 1) and cooled to room temperature (line 2) samples of GB and A series on the total surface area of a filler, $S W /(1-W)$. Vertical lines represent the width of experimental range of $\Delta H_{\mathrm{m}}{ }^{*}$ values obtained at different crystallization temperature.

3 in Figure 5a) are consistent with the assignment of this peak to the melting of $\gamma$-polymorph of a pure polymer, while slope $b(1)$ of nearly unity and the very low value of intercept $a(1)$ for line 1 in Figure 5a suggests that the low-temperature peak 1 is related to the melting of bundle-like crystals of nylon $6,{ }^{43}$ presumably formed at the latter stages of crystallization when lamellar growth is prohibited. ${ }^{39}$ Finally, near coincidence of line 2 for intermediate endo- thermic peak 2 , on one hand, with line 4 for melting temperature of low-crystallinity samples, on the other hand (Figure 5a), permits attributing the endothermic process 2 to the melting of an $\alpha$ polymorph of nylon $6^{32.41}$ It is pertinent to note here that although the latter conclusion tends to support Valenti et al.'s claim ${ }^{33}$ that the equlibrium melting temperature $T_{\mathrm{m}}{ }^{0}$ of crystalline $\alpha$-form of nylon 6 can be estimated from $T_{\mathrm{m}}(2) v s . T_{\mathrm{c}}$ plots, there are both theoretical and experimental reasons to believe that more reliable data can be obtained from $T_{\mathrm{m}}(4) v s . T_{\mathrm{c}}$ dependnce. ${ }^{34,35}$ Our value, $T_{\mathrm{m}}{ }^{0}=511 \pm 2 \mathrm{~K}$, for an $\alpha$ polymorph of pure nylon 6 , as determined from intersection of line 4 in Figure $5 \mathrm{a}$ with the "equilibrium" line $T_{\mathrm{m}}=T_{\mathrm{c}}$, is lower than either $518 \mathrm{~K}$ or $533 \mathrm{~K}$, reported by Arakawa et al. ${ }^{41}$ and Illers et al. ${ }^{31}$ respectively, but is very close to $T_{\mathrm{m}}{ }^{0}=513 \pm 2 \mathrm{~K}$ quoted by Valenti et al. ${ }^{33}$

Similarity between the melting patterns of pure nylon 6 and filled samples (Figures 4a, 4b, and 4c) seems to warrant the assumption that addition of a filler does not fundamentally change the molecular mechanisms responsible for multiple peaks on melting endotherms. As already mentioned above, the dependence of melting peaks $T_{\mathrm{m}}(1)$ and $T_{\mathrm{m}}$ (3) (if detectable) for all filled samples, on $T_{\mathrm{c}}$ is almost the same as that for a pure polymer. However, there is a substantial decrease of the slopes, $b(2)$ and $b(4)$ (and, consequently, of the equilibrium melting temperatures) for all samples containing untreated GB and $A$ fillers, except GB-50 ( $c f$. numerical data in Table I). The physical meaning of $b(4)$ ensues from the 
expression, ${ }^{43}$

$$
b(4)=0.5\left(l^{*} / l\right)\left(\sigma_{\mathrm{e}, \mathrm{l}} / \sigma_{\mathrm{e} . \mathrm{k}}\right)
$$

where $l^{*}$ and $l$ are the lamellar heights corresponding to the initial and final stages of crystallization, and $\sigma_{\mathrm{e}, \mathrm{k}}$ and $\sigma_{\mathrm{e}, 1}$ are surface free-energies of basal (i.e., fold-containing) planes of lamella estimated from the temperature dependence of crystallization rates, and from $T_{\mathrm{m}}$ dependence on $l$, respectively. In terms of eq 3 , experimentally observed $b(4)=0.538$ for pure nylon 6 implies, approximately, $l^{*} \cong l$ and $\sigma_{\mathrm{e}, \mathrm{l}} \cong \sigma_{\mathrm{e}, \mathrm{k}}$, which agrees with similar data for other unfilled polymers. ${ }^{43}$ On the other hand, taking into consideration the sudden drop of nucleation free energy (i.e., "kinetic" $\sigma_{\mathrm{e}, \mathrm{k}}$ ) for samples GB-70, GB$80, \mathrm{~GB}-83$, and $\mathrm{A}-3,{ }^{36}$ there are at least two alternative explanations for the concomitant decrease of $b(4)$, namely: 1, Lamellar thickening (i.e., $l>l^{*}$ ) which requires $\sigma_{\mathrm{e}, \mathrm{k}}=\sigma_{\mathrm{e}, 1} ; 2$, Smoothing of lamellar fold surfaces in the course of crystallization (i.e., $\left.\sigma_{\mathrm{e}, \mathrm{l}}<\sigma_{\mathrm{e}, \mathrm{k}}\right)$ at $l=l^{*}$. Evidently, special structural studies have to be undertaken in order to choose between these alternatives.

This argumentation can not be pursued further, however, to rationalize the experimentally found $b(4)=0$ for sample A-67. It appears rather more appropriate here to take into account the space limitations at extremely high filler loadings which should impose severe kinetic restrictions on lamellar growth in the course of crystallization, and presumably, bring about larger values of $\sigma_{e, k}$. In the case considered here, the minimum distance between surfaces of filler particles is approximately $30 \times 10^{-10} \mathrm{~m}$ (estimated, as $\mathrm{d}\left[\left(\varphi_{\mathrm{m}} / \varphi\right)^{1 / 3}-1\right]$, where $d=1.2 \times 10^{-8} \mathrm{~m}$ is the mean particle diameter, $\varphi_{\mathrm{m}} \cong 0.80$ and $\varphi=0.47$ are limiting (maximum) and actual volume contents of a filler). This value should be now compared with the theoretical height of a growth nucleus $l^{*}$ calculated from eq 4 below, viz. ${ }^{34,35,43}$ :

$$
l^{*} \cong 2 \sigma_{\mathrm{e}, \mathrm{k}} / \Delta g
$$

where $\Delta g=\Delta H_{\mathrm{m}} \Delta T / T_{\mathrm{m}}{ }^{0}$ is the bulk free-energy difference between polymer amorphous and crystalline states. Substitution of $\sigma_{\mathrm{e}, \mathrm{k}} \cong 60 \times 10^{-3} \mathrm{~J} \mathrm{~m}^{-2}, \quad 36$ $\Delta H_{\mathrm{m}}=176.4 \mathrm{~kJ} \mathrm{~kg}^{-1}=2.1 \times 10^{5} \mathrm{~kJ} \mathrm{~m}^{-3}$ and $T_{\mathrm{m}}{ }^{0}=$ $511 \mathrm{~K}$ (see above) into eq 4 yields $l^{*}=$ $95 \times 10^{-10} \mathrm{~m}$ at undercooling $\Delta T=30 \mathrm{~K}$ and $l^{*}=35 \times 10^{-10} \mathrm{~m}$ at $\Delta T=80 \mathrm{~K}$. These admittedly approximate calculations in which we did not allow for variations of $T_{\mathrm{m}}{ }^{0}$ or $\sigma_{\mathrm{e}, \mathrm{k}},{ }^{36}$ permit, nevertheless, explanation of a number of important experimental observations. To begin with, failure of sample A-67 to crystallize at $T_{\mathrm{c}}$ above $440 \mathrm{~K}^{36}$ turns out to be the consequence of the inability of a growth nucleus to reach its critical height, rather than lateral dimensions, $a^{*}$ or $b^{*}$, because, by definintion, ${ }^{34}$ $a^{*} \cong b^{*} \cong 2 \sigma / \Delta g$ (where $\sigma$ is the corresponding " $k$ inetic" surface free energy), and usually $\sigma<\sigma_{\mathrm{e}, \mathrm{k}}$. This conclusion is consistent with the universally recoginzed tendency of flexible-chain polymers to crystallize by a chain-folding mechanism with normal orientation of their long axes to the substrate surface. ${ }^{38,39}$ It follows, therefore, that for samples A50 and A-33, similar "critical" degrees of undercooling should exist, which the calculated values of $l^{*}$ match approximately the mean interparticle separations $\left(50 \times 10^{-10} \mathrm{~m}\right.$ and $90 \times 10^{-10} \mathrm{~m}$ respectively). The predicted values of "critical" $\Delta T$ 's $(65 \mathrm{~K}$ for A50 and $35 \mathrm{~K}$ for A-33) are again in a fair agreement with actual kinetic data. ${ }^{36}$ Moreover, space limitations for volume contraction during crystallization due to close proximity of neighboring solid particles seem to be a natural cause of internal stress build-up in highly loaded annealed samples of GB and the A series, one of manifestations of which are relaxation endotherms on $c_{p} v s$. $T$ plots above $T_{\mathrm{g}}$ (Figure 3 ).

The concept of internal stresses can also be used to explain the partial recovery of melting heats $\Delta H_{\mathrm{m}}{ }^{*}(2)$ of these samples cooled to room temperature (Figure 6). In fact, while in the case of a pure polymer this recovery, $\Delta h=\Delta H_{\mathrm{m}}{ }^{*}(2)-\Delta H_{\mathrm{m}}{ }^{*}(1)=$ $6 \mathrm{~kJ} \mathrm{~kg}^{-1}$, may be attributed to complemetary crystallization at low temperatures of a low-molecular weight fraction of our commercial, unfractionated sample, this argument apparently does not apply to rationalize the increase of $\Delta h$ with filler content. In our opinion, the different slopes of lines 1 and 2 in Figure 6 can be accounted for by the following physical interpretation. Energetic interactions between polymer molecules and solid phase to compensate for the surface energy of the latter would lead to a decrease in melt enthalpy, and consequently, in the enthalpy difference between amorphous and crystalline phases, that is, $\Delta H_{\mathrm{m}}$. Thus, slope $b^{\prime}(1)$ of the linear dependence of quasiequilibrium (isothermal) $\Delta H_{\mathrm{m}}{ }^{*}(1)$ values on the total solid surface area (line 1 in Figure 6) should have the physical meaning of filler surface energy, $H_{\mathrm{f}}$. Indeed, our value, $b^{\prime}(1)=185 \times 10^{-3} \mathrm{~J} \mathrm{~m}^{-2}$, lies well within 
the published range, $(160-220) \times 10^{-3} \mathrm{~J} \mathrm{~m}^{-2}$, of heats of wetting of untreated Aerosils by various polar liquids. ${ }^{44-47}$ On the other hand, since $H_{\mathrm{f}}$ is an intrinsic property of a solid, difference in slopes, $b^{\prime}(1)$ and $b^{\prime}(2)$, should be traced back to specific structural changes at polymer-filler interfaces, occurring during the cooling of the samples to room temperature. Taking into consideration the obvious fact that such cooling will not change the nature of interactions between polymer molecules and the solid surface, it appears that the lower value of $b^{\prime}(2)$ reflects the concomitant decrease of the total number of interaction sites. In other words, the thermodynamic driving force for polymer crystallization tends to free molecular segments "anchored" at filler surfaces, and pull them to the crystal growth faces. Thus, the thermodynamic state of a polymeric phase in highly filled samples at low and ambient temperatures should be vizualized as that of metastable equilibrium corresponding to a delicate balance between surface forces tending to minimize the total free energy of a system by increasing the solid surface coverage, on one hand, and bulk driving force for polymer crystallization which tends to increase the stability of initial crystalline phase by lowering its surface-to-volume ratio at the expense of the polymer-filler area of interaction, on the other. This model appears capable of accounting for all experimental findings discussed so far.

Finally, analysis of numerical values of the equilibrium melting temperatures $T_{\mathrm{m}}{ }^{0}$ of filled samples is in order. As already pointed out above, pure nylon 6 and samples of GBA series have approximately the same $T_{\mathrm{m}}{ }^{0}$, which should be attributable to the low surface energy of silanetreated glass beads. In fact, such treatment was reported to bring about almost a four-fold decrease of $H_{\mathrm{f}}{ }^{47} \mathrm{On}$ the other hand, for samples containing untreated GB and A fillers (except A-67, as discussed earlier), one observes an abrupt, reproducible drop from $T_{\mathrm{m}}{ }^{0}=512 \mathrm{~K}$ for GB-50 to $T_{\mathrm{m}}{ }^{0}=499 \mathrm{~K}$ for GB70 , after which $T_{\mathrm{m}}{ }^{0}$ remains approximately constant at $T_{\mathrm{m}}{ }^{0}=501 \pm 3 \mathrm{~K}$ (see Table I). We may note in passing that this behavior resembles a typical levelling-off of $T_{\mathrm{g}}$ in filled amorphous polymers above some "critical" filler content. ${ }^{9,10}$ Now, assuming that the "ideal" melting enthalpy, $\Delta H_{\mathrm{m}}$, exhibits the same dependence on filler content as the experimental heats of fusion, $\Delta H_{\mathrm{m}}{ }^{*}$ (Figure 6), it follows from the apparent constancy of $T_{\mathrm{m}}{ }^{0}\left(=\Delta H_{\mathrm{m}} / \Delta S_{\mathrm{m}}\right)$ that the melting entropy, $\Delta S_{\mathrm{m}}$, should also be a linear function of the total filler surface area, $S W /(1-W)$. But this conclusion is not inconsistent with the above discussion, since energetic interactions between macromolecules and the solid surface should necessarily decrease the conformational freedom of the former, i.e., their entropy. However, one must assume that some discontinuous structural change occurs in the samples of the GB series in the range of filler loadings, $50<W<70 \quad$ (i.e., in the "critical" range of mean interparticle distances, $5 \times 10^{-6} \mathrm{~m}<$ $x_{\text {crit }}<10 \times 10^{-6} \mathrm{~m}$, to explain the initial drop of $T_{\mathrm{m}}{ }^{0}$. It turns out that the postulated change should involve a specific molecular mechanism which would bring about either a decrease in the melting enthalpy, $\Delta H_{\mathrm{m}}$, or an increase in the melting entropy, $\Delta S_{\mathrm{m}}$, of sample GB-70 over that of GB-50. The first suggestion appears unlikely in this case because the only plausible source of $\Delta H_{\mathrm{m}}$ change, that is, dissipation of crystallization heat on polymer-filler interface, should be extremely small at close-to-zero values of the total interfacial areas involved, since the specific surface area of glass beads is at least five orders of magnitude smaller than that of Aerosil 200. On the other hand, a slight increase in $\Delta S_{\mathrm{m}}$ resulting from a (partial) breakdown of hydrogen-bonded, ordered structures in nylon 6 melt, may be conceived as a transition of all polymer phases into boundary layers and is completed above a "critical" filler content. Unfortunately, at this stage, we can not completely assess the relevance of a "critical" distance between filler particles, $x_{\text {crit }}=(5$ 10) $\times 10^{-6} \mathrm{~m}$, below which all polymers apparently become involved in the "boundary state" different from melt structure of nylon 6 (see, however, part II, ref 36), although we deduced a similar value from thermodynamic studies of polystyrene melts filled with untreated glass powder. ${ }^{7}$ Further work is needed to elucidate the dependence of $x_{\text {crit }}$ on the structural characteristics of polymer melts.

\section{CONCLUSIONS}

The results of this study have shown that the thermodynamic state of a polymeric component in filled nylon 6 is controlled by energetic characteristics of the solid surface. Low-energy filler does nor affect in any appreciable way the melting parameters of a polymer; but both the equilibrium melting temperature and melting enthalpy. were found to 
undergo drastic changes as the thickness of a polymeric layer between high-energy solid particles decreased below $x_{\text {crit }}=(5-10) \times 10^{-6} \mathrm{~m}$. On the basis of our experimental data, we can offer the following qualitative model of polymer-solid interactions in samples containing untreated GB and A fillers. In the range of low-to-moderate filler contents (i.e., at mean interparticle separations above $x_{\text {crit }}$, while polymer adsorption on filler surface would undoubtedly result in appearance of boundarypolymer layers, the latter do not form a continuous network, however, and remaining "free" polymer retains essentially the melting characteristics of an initial, unfilled polymer. On the other hand, the transition of all polymer phases into the "boundary state" as interparticle distance decreases below $x_{\text {crit }}$, apparently destroys the initial melt structure, slightly increasing thereby its entropy and decreasing the equlibrium melting temperature, as well as creating favorable conditions for crystal nucleation. However, at higher filler loadings the solid phase begins to act both as a thermodynamic."sink" which absorbs heat (and, presumably, entropy) of crystallization, as well as a steric obstacle to crystal nucleation and growth. Consequently, the experimentally feasible range of crystallization temperatures becomes narrower and shifts to lower temperatures. Moreover, competition between surface forces tending to increase the number of polymer-filler interaction sites, and bulk driving force for crystallization and lamellar perfection which tends to pull macromolecules from filler surface onto crystal face, results in a gigantic build-up of internal stresses in highly filled samples cooled to and below room temperature, and these are shown as large relaxation endotherms on heat capacity curves above the glasstransition interval. Clearly, the importance of these relaxation phenomena should be realized both by polymer scientists studying the physical properties of filled polymers, as well as by potential users of filled polymeric composites.

\section{Acknowledgment.}

Thanks are due to Dr. T. Komoto and Mr. Ch. Sawada of Tokyo Institute of technology for their expert assistance at an early stage of this experimental work. The invitation extended to one of us (V.P.P) by the Japanese Society for the Promotion of Science on the "Visiting Professorship Program" for 1978 is also greatfully acknowledged.

\section{REFERENCES}

1. Yu. S. Lipatov and G. M. Pavlyuchenko, Vysokomol. Soedin., Ser. A, 2, 1564 (1960).

2. C. Kumins and J. Roteman, J. Polym. Sci. A, 1, 527 (1963).

3. T. K. Kwei, J. Polym. Sci. A, 3, 3229 (1965).

4. D. Droste and A. T. Dibenedetto, J. Appl. Polym. Sci., 13, 2149 (1969).

5. V. F. Babich and Yu. S. Lipatov, Mekh. Polim., 3, 548 (1969).

6. T. Kajiyama, T. Yoshinaga, and M. Takayanagi, $J$. Polym. Sci., Polym. Phys. Ed., 15, 1557 (1977).

7. V. P. Privalko, Yu. D. Besklubenko, Yu. S. Lipatov, S. S. Demchenko, and G. I. Khmelenko, Vysokomol. Soedin., Ser. A, 19, 1744 (1977).

8. K. Iisaka and K. Shibayama, J. Appl. Polym. Sci., 22, 1321 (1978).

9. Yu. S. Lipatov, "Physical Chemistry of Filled Polymers," Izdvestbo Khimia, Moscow, 1977 (in Russian).

10. Yu. S. Lipatov and V. P. Privalko, Vysokomol. Soedin., Ser. A, 14, 1643 (1972).

11. Yu. M. Malinskii, Usp. Khim., 39, 1511 (1970).

12. Yu. S. Lipatov and F. G. Fabulyak, J. Appl. Polym. Sci., 10, 2131 (1972).

13. Yu. S. Liṕatov, V. P. Privalko, Yu. D. Besklubenko, and S. S. Demchenko, Vysokomol. Soedin., Ser. A, 19, 1756 (1977).

14. Yu. S. Lipatov, Adv. Polym. Sci., 22, 1 (1977).

15. S. S. Krafchik, Yu. Yu. Kercha, and Yu. S. Lipatov, Sintez Fiz. Khim. Polimerov, No. 8, 103 (1971).

16. G. J. Howard, Kolloid Z. Z. Polym., 244, 213 (1971).

17. V. P. Privalko, G. I. Khmelenko, and Yu. S. Lipatov, Sintez Fiz. Khim. Polimerov, No. 16, 107 (1975).

18. V. A. Kargin, T. I. Sogolova, and I. I. Kurbanova, Dokl. Akad. Nauk. USSR, 162, 1092 (1965).

19. H. N. Beck and H. D. Ledbetter, J. Appl. Polym. Sci., 9, 2131 (1965).

20. M. Inoue, J. Polym. Sci., A, 1, 2013 (1963).

21. F. L. Binsbergen, Polymer, 11, 253 (1970).

22. G. Groeninckx, H. Bergmans, N. Overbergh, and G. Smets, J. Polym. Sci., Polym. Phys. Ed., 12, 303 (1974).

23. I. Gutzov, V. Dochev, E. Pancheva, and K. Dimov, J. Polym. Sci., Polym. Phys. Ed., 16, 1155 (1978).

24. G. Gurato, D. Gaidano, and R. Zannetti, Makromol. Chem., 179, 231 (1978).

25. E. P. Chang, R. O. Kirsten, and E. L. Slagowski, Polym. Eng. Sci., 18, 932 (1978).

26. S. Gogolewski and A. J. Pennings, Polymer, 16, 673 (1975).

27. P. Engler and S. H. Carr, Polym. Eng. Sci., 18, 450 (1978).

28. DSC-1 Instruction Manual, Perkin-Elmer Corp., Norwalk, Conn., 1969.

29. V. Bares and B. Wunderlich, J. Polym. Sci., Polym. Phys. Ed., 11, 861 (1973). 
30. D. R. Gee and T. P. Melia, Polymer, 11, 192 (1970).

31. K.-H. Illers and H. Haberkorn, Makromol. Chem., 142, 31 (1971).

32. G. Gurato, A. Fichera, F. Z. Grandi, R. Zannetti, and P. Canal, Makromol. Chem., 175, 953 (1974).

33. B. Valenti, G. Greppi, A. Tealdi, and A. Ciferri, $J$. Phys. Chem., 77, 389 (1973).

34. J. D. Hoffman, SPE Trans., 4, 315 (1964).

35. M. Gopalan and L. Mandelkern, J. Phys. Chem., 71, 3833 (1967).

36. V. P. Privalko, T. Kawai and Yu. S. Lipatov, Colloid Polym. Sci. (Part II, submitted for publication).

37. G. I. Tsereteli, Thesis, Leningrad State University, Leningrad, 1975.

38. B. Wunderlich, "Macromolecular Physics", Academic Press, New York, 1976, Vol. II.

39. R. Chujo and T. Kawai, "Physical Chemistry of Polymers", Kyoritsu Shuppan Publ. Co., Tokyo,
1973 (in Japanese).

40. F. Danusso, Polymer, 8, 281 (1967).

41. T. Arakawa, F. Nagatoshi, and N. Arai, J. Polym. Sci., A-2, 7, 1461 (1969).

42. M. Kyotani and S. Mitsuhashi, J. Polym. Sci., A-2, 10, 1497 (1972).

43. T. Matsumoto, N. Ikegami, K. Ehara, T. Kawai, and H. Maeda, Kogyo Kagaku Zasshi, 73, 2441 (1970).

44. M. M. Egorov, K. G. Krasil'nikov, and E. A. Sysoev, Dokl. Akad. Nauk USSR, 108, 103 (1956).

45. A. C. Zettlemoyer, J. J. Chessick, and C. M. Hollabaugh, J. Phys. Chem., 62, 489 (1958).

46. A. C. Makrides and N. Hackerman, J. Phys. Chem., 63, 594 (1959).

47. H. Utsugi and S. Nishimura, Nihon Kagaku Zasshi, 92, 759 (1971).

48. S. K. Rhee, Mater. Sci. Eng., 19, 151 (1975). 